\title{
Can One Use Mueller-Navelet Jets at LHC as a Clean Test of QCD Resummation Effects at High Energy? $?^{\S}$
}

\author{
Bertrand Ducloué ${ }^{1}$, Lech Szymanowski ${ }^{2}$ and Samuel Wallon ${ }^{*}, 1,3$ \\ ${ }^{1}$ LPT, Université Paris-Sud, CNRS, 91405, Orsay, France \\ ${ }^{2}$ National Centre for Nuclear Research (NCBJ), Warsaw, Poland \\ ${ }^{3}$ UPMC Univ. Paris 06, Faculté de physique, 4 place Jussieu, 75252 Paris Cedex 05, France
}

\begin{abstract}
The measurement of azimuthal correlations of Mueller-Navelet jets is generally considered as a decisive test to reveal the effect of BFKL dynamics at hadron colliders. The first experimental study of these correlations at the LHC has been recently performed by the CMS collaboration. We show that the ratios of cosine moments of the azimuthal distribution are successfully described within our next-to-leading logarithmic BFKL treatment. The whole set of CMS data for the azimuthal correlations can also be consistently described, provided that one uses a larger renormalization/factorization scale than its natural value.
\end{abstract}

PACS Numbers: 12.38.Cy, 12.38.Qk, 13.85.Hd.

Keywords: Collider physics, high-energy resummation, jets, LHC, QCD.

\section{INTRODUCTION}

The understanding of the high energy limit of QCD, in the so-called perturbative Regge limit, has been the subject of many studies. Many observables have been suggested to test these dynamics, based on inclusive [1], semi-inclusive [2] and exclusive processes [3]. In this limit, the smallness of the strong coupling $\alpha_{s}$ can be compensated by large logarithmic enhancements of the type $\left[\alpha_{s} \ln (s /|t|)\right]^{n}$ which have to be resummed, giving rise to the leading logarithmic (LL) Balitsky-Fadin-Kuraev-Lipatov (BFKL) Pomeron [4]. Mueller and Navelet proposed to study the production of two jets with a large rapidity separation at hadron colliders [5]. In a pure leading order collinear treatment, these two jets would be emitted back to back, while a BFKL approach allows some emission between these jets which should lead to a larger cross section and lower angular correlation of the jets. We present results of a full next-to-leading logarithmic (NLL) analysis, in which the NLL corrections are included for the BFKL Green's function [6] and the jet vertices [7, 8].

In the following we will focus on the azimuthal correlations $\langle\cos n \varphi\rangle$ [9] and ratios of these observables, as well as on the azimuthal distribution, at a center of mass energy $\sqrt{s}=7 \mathrm{TeV}$, which have been measured recently at the LHC by the CMS collaboration [10]. We make some

*Address correspondence to this author at the LPT, Université Paris-Sud, CNRS, 91405, Orsay, France; Fax: 33 (0) 1691582 87;

E-mail: Samuel.Wallon@th.u-psud.fr

${ }^{\S}$ Presented at the Low x workshop, May 30 - June 4 2013, Rehovot and Eilat, Israel. comparison of our results [11] with these data and investigate the dependency on the various scales, including the renormalization scale.

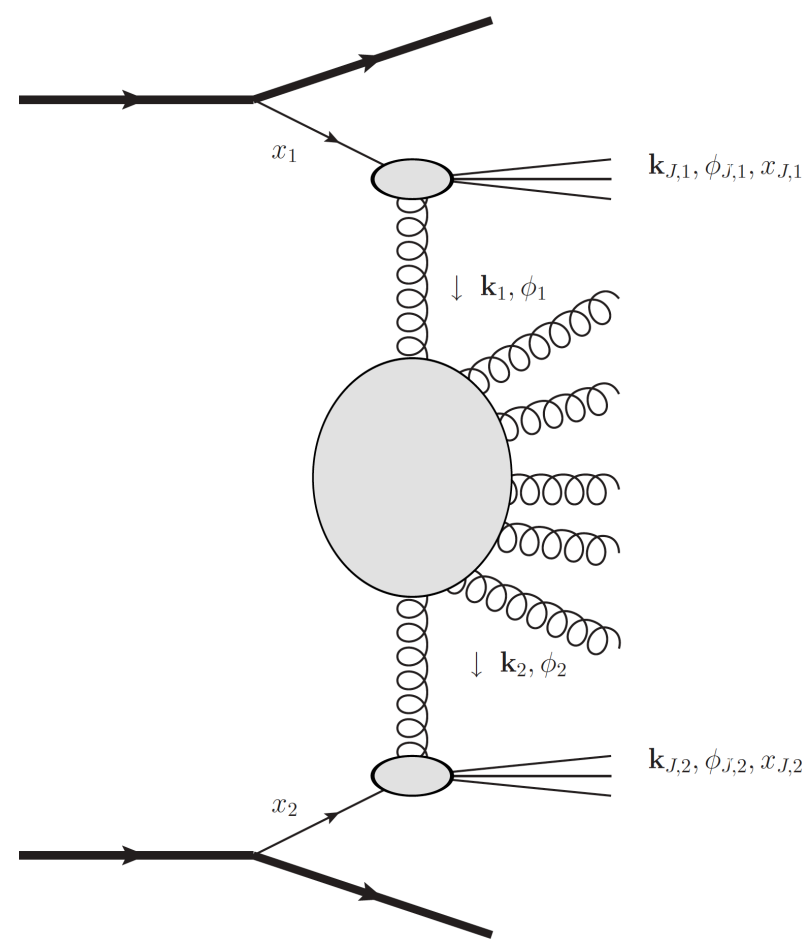

Fig. (1). Kinematics of the process.

\section{BASIC FORMULAS}

Let us consider, as shown on Fig. (1), two hadrons colliding at a center of mass energy $\sqrt{s}$. Relying on the collinear factorization, the differential cross section reads 


$$
\begin{aligned}
& \frac{\mathrm{d} \sigma}{\mathrm{d}\left|\mathbf{k}_{J, 1}\right| \mathrm{d}\left|\mathbf{k}_{J, 2}\right| \mathrm{d} y_{J, 1} \mathrm{~d} y_{J, 2}} \\
& =\sum_{\mathrm{a}, \mathrm{b}} \int_{0}^{1} \mathrm{~d} x_{1} \int_{0}^{1} \mathrm{~d} x_{2} f_{\mathrm{a}}\left(x_{1}\right) f_{\mathrm{b}}\left(x_{2}\right) \frac{\mathrm{d} \hat{\sigma}_{\mathrm{ab}}}{\mathrm{d}\left|\mathbf{k}_{J, 1}\right| \mathrm{d}\left|\mathbf{k}_{J, 2}\right| \mathrm{d} y_{J, 1} \mathrm{~d} y_{J, 2}},
\end{aligned}
$$

where $\mathbf{k}_{J, 1}, \mathbf{k}_{J, 2}$ are the transverse momenta of the jets, $y_{j, 1}$ and $y_{j, 2}$ their rapidities and $f_{a}\left(f_{b}\right)$ are the parton distribution functions (PDFs) of a parton a (b) in the according proton. In this expression, the partonic cross section can be expressed as

$$
\begin{aligned}
& \frac{\mathrm{d} \hat{\sigma}_{\mathrm{ab}}}{\mathrm{d}\left|\mathbf{k}_{J, 1}\right| \mathrm{d}\left|\mathbf{k}_{J, 2}\right| \mathrm{d} y_{J, 1} \mathrm{~d} y_{J, 2}} \\
& =\int \mathrm{d} \phi_{J, 1} \mathrm{~d} \phi_{J, 2} \int \mathrm{d}^{2} \mathbf{k}_{1} \mathrm{~d}^{2} \mathbf{k}_{2} V_{\mathrm{a}}\left(-\mathbf{k}_{1}, x_{1}\right) G\left(\mathbf{k}_{1}, \mathbf{k}_{2}, \hat{s}\right) V_{\mathrm{b}}\left(\mathbf{k}_{2}, x_{2}\right),
\end{aligned}
$$

where $\phi_{J, 1}$ and $\phi_{J, 2}$ are the azimuthal angles of the jets, $V_{a}\left(V_{b}\right)$ is the jet vertex initiated by the parton a (b) and $G$ is the BFKL Green's function which depends on $\hat{s}=x_{1} x_{2} s$. For further use, it is convenient to introduce the coefficients $\mathcal{C}_{n}$, defined as

$$
\mathcal{C}_{n}=\left(4-3 \delta_{n, 0}\right) \int \mathrm{d} v C_{n, v}\left(\left|\mathbf{k}_{J, 1}\right|, x_{J, 1}\right) C_{n, v}^{*}\left(\left|\mathbf{k}_{J, 2}\right|, x_{J, 2}\right)\left(\frac{\hat{s}}{s_{0}}\right)^{\omega(n, v)},
$$

such that

$$
\frac{\mathrm{d} \sigma}{\mathrm{d}\left|\mathbf{k}_{J, 1}\right| \mathrm{d}\left|\mathbf{k}_{J, 2}\right| \mathrm{d} y_{J, 1} \mathrm{~d} y_{J, 2}}=\mathcal{C}_{0},
$$

and

$$
\langle\cos (n \varphi)\rangle \equiv\left\langle\cos \left(n\left(\phi_{J, 1}-\phi_{J, 2}-\pi\right)\right)\right\rangle=\frac{\mathcal{C}_{n}}{\mathcal{C}_{0}} .
$$

In Eq. (3), $C_{n, v}$ is defined as

$$
C_{n, v}\left(\left|\mathbf{k}_{J}\right|, x_{J}\right)=\int \mathrm{d} \phi_{J} \mathrm{~d}^{2} \mathbf{k} \mathrm{d} x f(x) V(\mathbf{k}, x) E_{n, v}(\mathbf{k}) \cos \left(n \phi_{J}\right),
$$

with $E_{n, v}$ being the LL BFKL eigenfunctions

$$
E_{n, v}\left(\mathbf{k}_{1}\right)=\frac{1}{\pi \sqrt{2}}\left(\mathbf{k}_{1}^{2}\right)^{i v-\frac{1}{2}} e^{i n \phi_{1}}
$$

At LL accuracy, the eigenvalue $\omega(n, v)$ of the BFKL kernel is

$$
\omega(n, v)=\bar{\alpha}_{s} \chi_{0}\left(|n|, \frac{1}{2}+i v\right)
$$

with

$$
\chi_{0}(n, \gamma)=2 \Psi(1)-\Psi\left(\gamma+\frac{n}{2}\right)-\Psi\left(1-\gamma+\frac{n}{2}\right)
$$

where $\bar{\alpha}_{s}=\alpha_{s} N_{c} / \pi, \Psi(z)=\Gamma^{\prime}(z) / \Gamma(z)$ and the vertex is
$V_{\mathrm{a}}(\mathbf{k}, x)=V_{\mathrm{a}}^{(0)}(\mathbf{k}, x)=\frac{\alpha_{s}}{\sqrt{2}} \frac{C_{A / F}}{\mathbf{k}^{2}} \delta\left(1-\frac{x_{J}}{x}\right)\left|\mathbf{k}_{J}\right| \delta^{(2)}\left(\mathbf{k}-\mathbf{k}_{J}\right)$,

with $C_{A}$ for $\mathrm{a}=\mathrm{g}$ and $C_{F}$ for $\mathrm{a}=\mathrm{q}$. At NLL, despite the fact that the $E_{n, v}$ are not eigenfunctions of the kernel anymore due to conformal invariance breaking terms, it is still possible to use them, the price to pay being an explicit dependency on $\mathbf{k}_{J, 1}$ and $\mathbf{k}_{J, 2}[12-14]$ :

$$
\begin{aligned}
& \omega(n, v)=\bar{\alpha}_{s} \chi_{0}\left(|n|, \frac{1}{2}+i v\right) \\
& +\bar{\alpha}_{s}^{2}\left[\chi_{1}\left(|n|, \frac{1}{2}+i v\right)-\frac{\pi b_{0}}{N_{c}} \chi_{0}\left(|n|, \frac{1}{2}+i v\right) \ln \frac{\left|\mathbf{k}_{J, 1}\right| \cdot\left|\mathbf{k}_{J, 2}\right|}{\mu_{R}^{2}}\right],
\end{aligned}
$$

with $b_{0}=\left(33-2 N_{f}\right) /(12 \pi)$ and $V_{\mathrm{a}}(\mathbf{k}, x)=V_{\mathrm{a}}^{(0)}(\mathbf{k}, x)+\alpha_{s} V_{\mathrm{a}}^{(1)}(\mathbf{k}, x)$. The expression for the NLL corrections to the Green's function resulting in $\chi_{1}$ can be found in Eq. (2.17) of [11]. The expressions of the NLL corrections to the jet vertices are quite lengthy and will not be reproduced here. They can be found in [15], as extracted from [7] after correcting a few misprints. They have been recently reobtained in [8]. In the limit of small cone jets, they have been computed in [16] and applied to phenomenology in $[17,18]$. Here we use the cone algorithm with a size of $R_{\text {cone }}=0.5$. Note, however, that using the $k_{t}$ or the anti- $k_{t}$ algorithm leads to negligible changes in our predictions. Our calculation depends on the renormalization scale $\mu_{R}$, the factorization scale $\mu_{F}$ and the energy scale $\sqrt{s_{0}}$. In the following we set $\mu_{R}=\mu_{F} \equiv \mu$. We choose the "natural" value $\sqrt{\left|\mathbf{k}_{J, 1}\right| \cdot\left|\mathbf{k}_{J, 2}\right|}$ for $\mu$ and $\sqrt{s_{0}}$, and vary these scales by a factor of 2 to estimate the scale uncertainty of our calculation. We use the MSTW 2008 PDFs [19] and a two-loop running coupling. We also include collinear improvement to the Green's function as was suggested in [20] and extended for $n \neq 0$ in [13, 14, 21].

\section{RESULTS: ASYMMETRIC CONFIGURATION}

In [11] we performed a detailed study of several BFKL scenarios, from a pure LL approximation (LL Green's function and leading order jet vertex) to a full NLL calculation (NLL Green's function and NLL jet vertex). The main conclusion is that there is a dramatic effect when passing from a mixed treatment, which combines LL vertices with NLL Green's function, to a full NLL approach, of magnitude similar to the one when passing from a pure LL result to the mixed treatment; the inclusion of NLL corrections inside the jets vertices is therefore essential. Note that the effect of collinear improvement in the NLL Green's function is very small when using the NLL jet vertices. The inclusion of the whole set of NLL corrections leads to decorrelation effects which are much smaller than expected. Still, there remains a sizable difference between the full NLL approach and the fixed order predictions at next-to-leading order (NLO) for the ratios $\langle\cos 2 \varphi\rangle /\langle\cos \varphi\rangle$ and $\langle\cos 3 \varphi\rangle /\langle\cos 2 \varphi\rangle$, when taking into account theoretical uncertainties. 
To study the need for high-energy resummation with respect to fixed order treatments, one should, however, pay attention to the fact that these fixed order calculations have instabilities when the lower cuts on the transverse momenta of the jets are identical. Thus, at the moment, it is not possible to make a direct comparison between these two predictions in an asymmetric configuration and the data of [10], which have been extracted in a symmetric configuration. Still, one can compare our BFKL calculation with the fixed order NLO code Dijet [22] in an asymmetric configuration with the following cuts, which could be implemented by CMS:

$$
\begin{aligned}
& 35 \mathrm{GeV}<\left|\mathbf{k}_{J, 1}\right|,\left|\mathbf{k}_{J, 2}\right|<60 \mathrm{GeV}, \\
& 50 \mathrm{GeV}<\operatorname{Max}\left(\left|\mathbf{k}_{J, 1}\right|,\left|\mathbf{k}_{J, 2}\right|\right), \\
& 0<\quad y_{1}, y_{2} \quad<4.7 .
\end{aligned}
$$

This is illustrated for the ratios $\langle\cos 2 \varphi\rangle /\langle\cos \varphi\rangle$ and $\langle\cos 3 \varphi\rangle /\langle\cos 2 \varphi\rangle$ in Fig. (2). In this figure, we show the variation of our NLL result when varying $\mu$ and $\sqrt{s_{0}}$ by a factor of 2 and compare it with the Dijet prediction. Here the fixed order NLO calculation is significantly above the full NLL BFKL calculation. These observables are quite stable with respect to the scales so that the difference between NLL BFKL and fixed order NLO does not vanish when we take into account the scale uncertainty.

\section{RESULTS: SYMMETRIC CONFIGURATION}

In this section, we compare our results with the data recently obtained by CMS [10], for a symmetric configuration (identical lower cut for the transverse momenta of the jets) and with cuts

$$
\begin{aligned}
& 35 \mathrm{GeV}<\left|\mathbf{k}_{J, 1}\right|,\left|\mathbf{k}_{J, 2}\right|<60 \mathrm{GeV}, \\
& 0<\quad y_{1}, y_{2}<4.7 .
\end{aligned}
$$

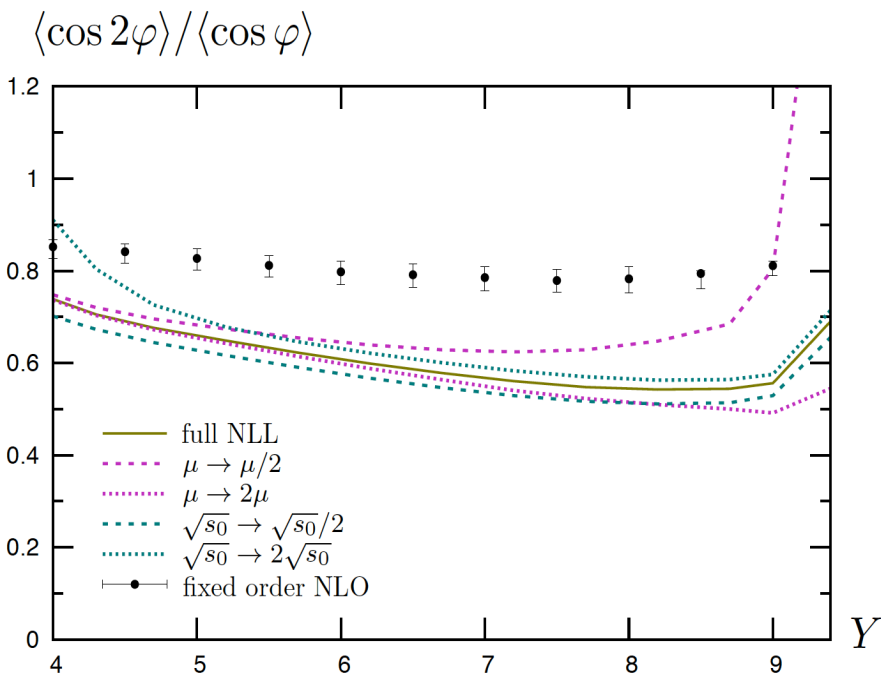

These are the cuts used by CMS in [10], with the exception that for numerical reasons we have to set an upper cut on the transverse momenta of the jets. We have checked that our results do not depend strongly on the value of this cut as the cross section is strongly peaked near the minimum value allowed for $\mathbf{k}_{J, 1}$ and $\mathbf{k}_{J, 2}$. This enables us to compare our predictions with LHC data.

We begin our analysis with the azimuthal correlations $\langle\cos n \varphi\rangle$. In Figs. $(\mathbf{3}, \mathbf{4})$ we show the variation of $\langle\cos \varphi\rangle$, $\langle\cos 2 \varphi\rangle$ and $\langle\cos 3 \varphi\rangle$ with respect to the rapidity separation between the two jets $Y$ within a full NLL framework. We display the theoretical uncertainty obtained when varying $\mu$ and $s_{0}$ by a factor of 2 and we compare these predictions with CMS data (black dots with error bars). We see that NLL BFKL predicts a larger correlation than seen in the data, but these observables are strongly dependent on the value of the scales.

The extraction of ratios of the previously mentioned observables was also performed in [10]. In Fig. (5) we show results for $\langle\cos 2 \varphi\rangle /\langle\cos \varphi\rangle$ and $\langle\cos 3 \varphi\rangle /\langle\cos 2 \varphi\rangle$. The effect of NLL corrections to the vertices is important (see $[11,23]$ for detailed comparisons), but these observables are more stable with respect to $\mu$ and $s_{0}$ than the previous ones. The agreement with the data is very good over a large $Y$ range.

Our code allows us to perform a complete study of the azimuthal distribution [24], which is most directly accessible in experiments, while more difficult to evaluate numerically than the individual moments discussed above. It is defined as

$$
\frac{1}{\sigma} \frac{d \sigma}{d \varphi}=\frac{1}{2 \pi}\left\{1+2 \sum_{n=1}^{\infty} \cos (n \varphi)\langle\cos (n \varphi)\rangle\right\} \text {. }
$$

If one tries to compare the full NLL BFKL prediction with CMS data, we fail to describe the distribution for large values of $\varphi$, corresponding to nearside jets configurations,

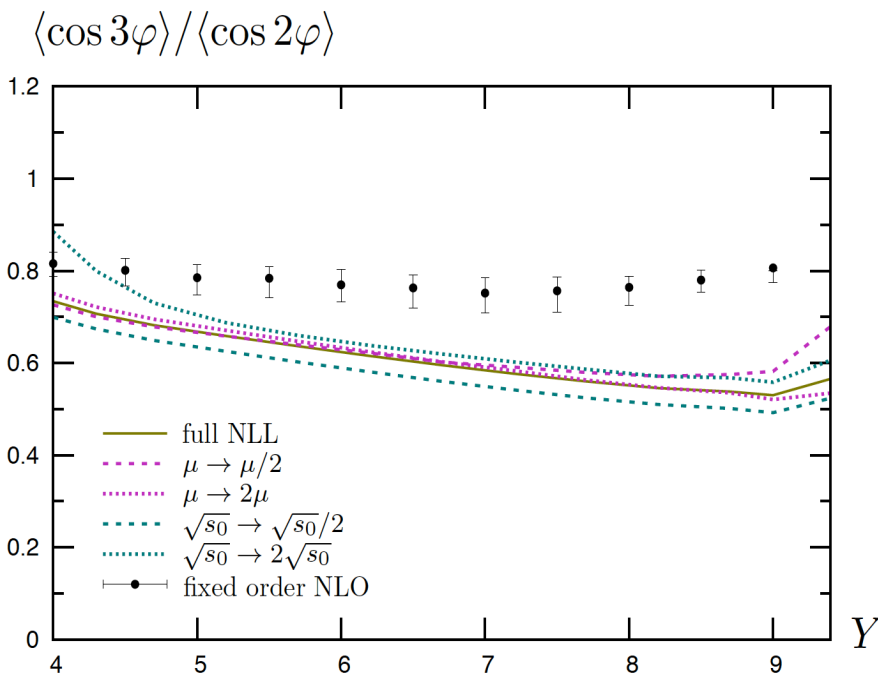

Fig. (2). Comparison of the full NLL BFKL calculation including the scale uncertainty with Dijet predictions, using asymmetric cuts defined in Eq. (12). Left: value of $\langle\cos 2 \varphi\rangle /\langle\cos \varphi\rangle$ as a function of the rapidity separation $Y$. Right: value of $\langle\cos 3 \varphi\rangle /\langle\cos 2 \varphi\rangle$ as a function of the rapidity separation $Y$. 
$\langle\cos \varphi\rangle$

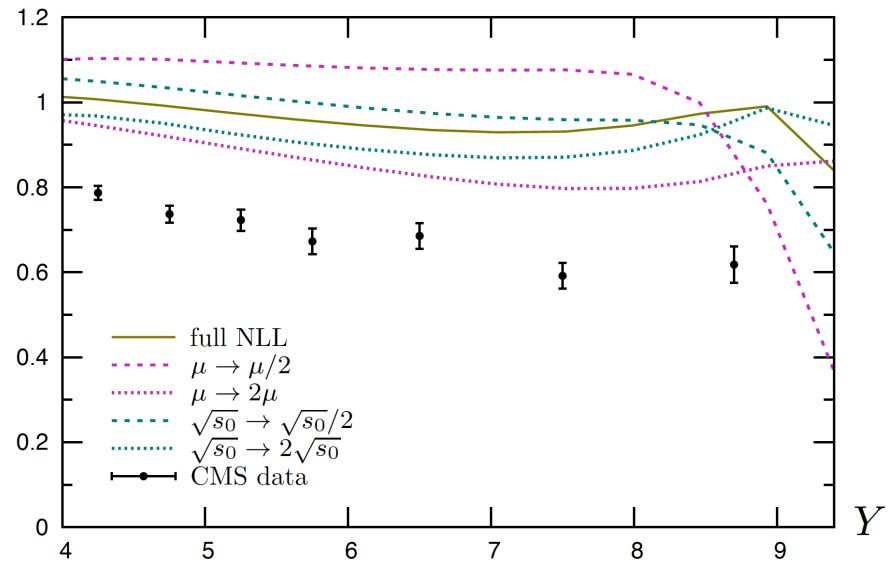

$\langle\cos 2 \varphi\rangle$

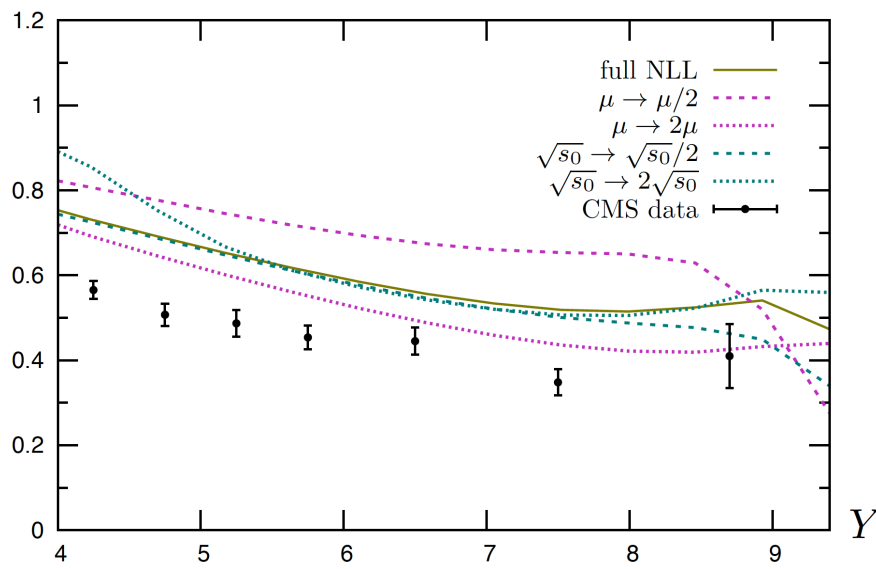

Fig. (3). Comparison of the full NLL BFKL calculation including the scale uncertainty with CMS data, using symmetric cuts defined in Eq. (13). Left: value of $\langle\cos \varphi\rangle$ as a function of the rapidity separation $Y$. Right: value of $\langle\cos 2 \varphi\rangle$ as a function of the rapidity separation $Y$.

when taking a "natural" scale $\mu=\sqrt{\left|\mathbf{k}_{J, 1}\right| \cdot\left|\mathbf{k}_{J, 2}\right|}$. We have seen also in Figs. $(3,4)$ that the data is better described if we use larger values of $\mu$. Indeed, if we let the renormalization/factorization scale vary by more than a factor of 2, one can see that the whole set of CMS data can be very well described within the full NLL BFKL approach with a scale of the order of $\mu \sim 8 \sqrt{\left|\mathbf{k}_{J, 1}\right| \cdot\left|\mathbf{k}_{J, 2}\right|}$. This is illustrated in Figs. (6-8).

\section{CONCLUSION}

We have been able to compare the predictions of our full NLL BFKL calculation of Mueller-Navelet jets with data taken at the LHC thanks to the measurement presented by the CMS collaboration. This comparison shows [23] that for the observables $\langle\cos n \varphi\rangle$ a pure LL BFKL treatment or a

$\langle\cos 2 \varphi\rangle /\langle\cos \varphi\rangle$

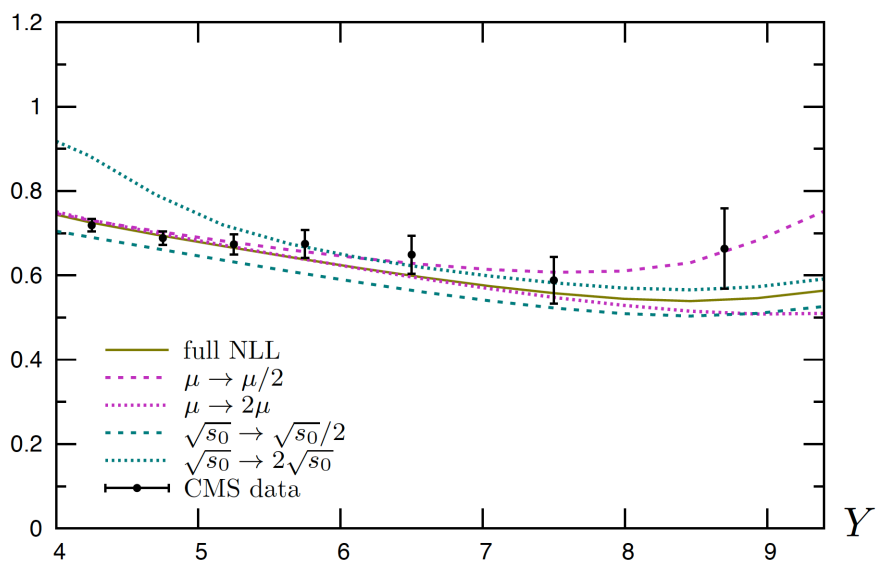

$\langle\cos 3 \varphi\rangle$

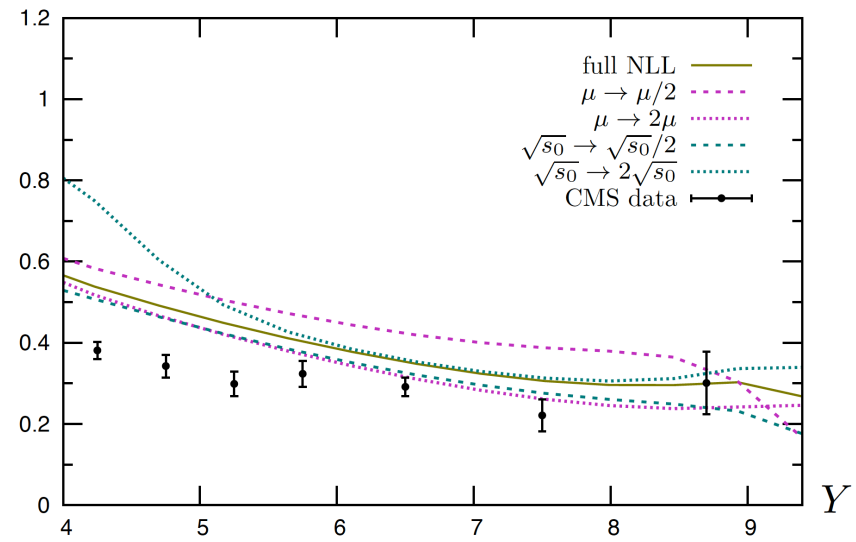

Fig. (4). Comparison of the full NLL BFKL calculation including the scale uncertainty with CMS data, using symmetric cuts defined in Eq. (13), for $\langle\cos 3 \varphi\rangle$ as a function of the rapidity separation $Y$.

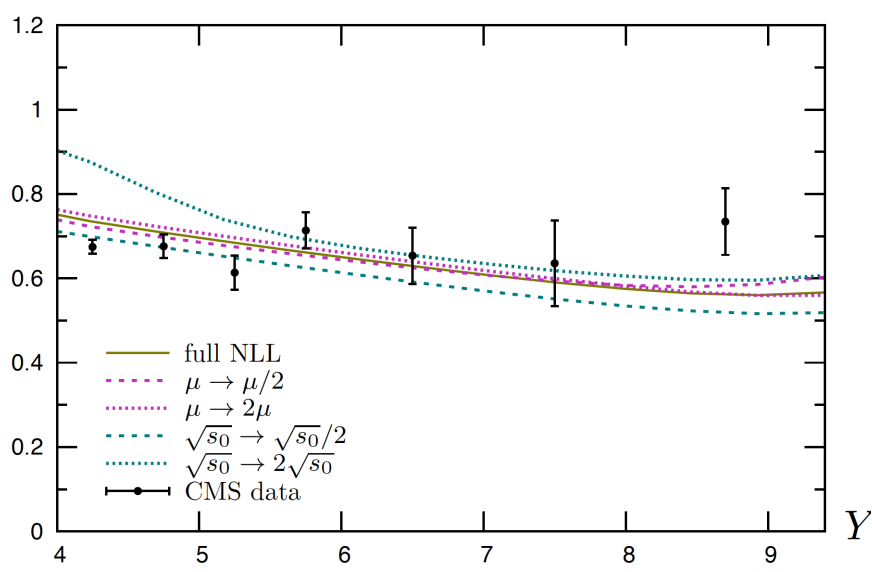

Fig. (5). Comparison of the full NLL BFKL calculation including the scale uncertainty with CMS data, using symmetric cuts defined in Eq. (13). Left: value of $\langle\cos 2 \varphi\rangle /\langle\cos \varphi\rangle$ as a function of the rapidity separation $Y$. Right: value of $\langle\cos 3 \varphi\rangle /\langle\cos 2 \varphi\rangle$ as a function of the rapidity separation $Y$. 
$\langle\cos \varphi\rangle$

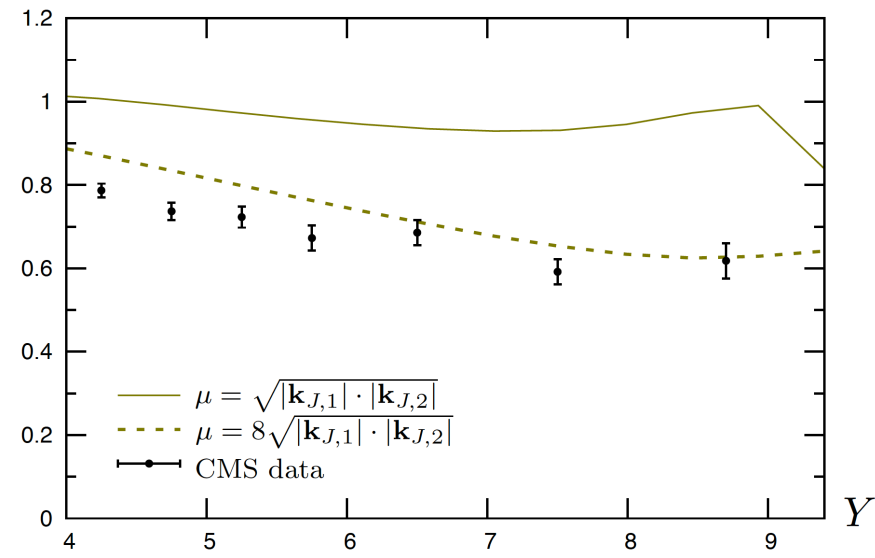

$\langle\cos 2 \varphi\rangle$

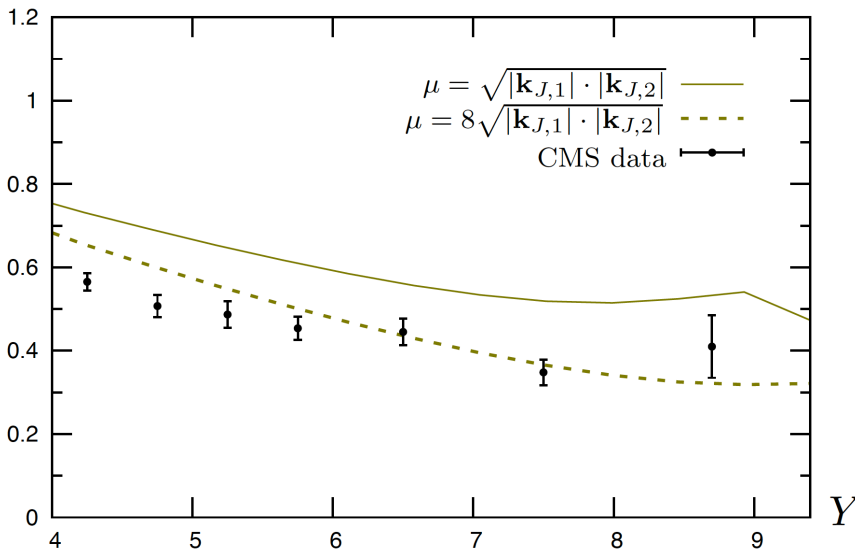

Fig. (6). Comparison of the full NLL BFKL calculation with either a "natural" scale $\mu=\sqrt{\left|\mathbf{k}_{J, 1}\right| \cdot\left|\mathbf{k}_{J, 2}\right|}$ or a large scale $\mu=8 \sqrt{\left|\mathbf{k}_{J, 1}\right| \cdot\left|\mathbf{k}_{J, 2}\right|}$ and the CMS data, using symmetric cuts defined in Eq. (13). Left: value of $\langle\cos \varphi\rangle$ as a function of the rapidity separation $Y$. Right: value of $\langle\cos 2 \varphi\rangle$ as a function of the rapidity separation $Y$.

$\langle\cos 3 \varphi\rangle$

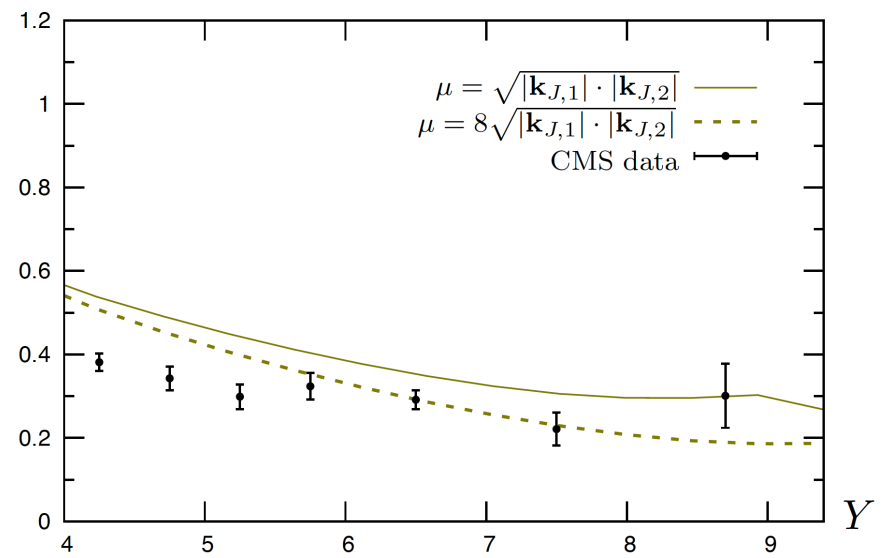

$\langle\cos 2 \varphi\rangle /\langle\cos \varphi\rangle$

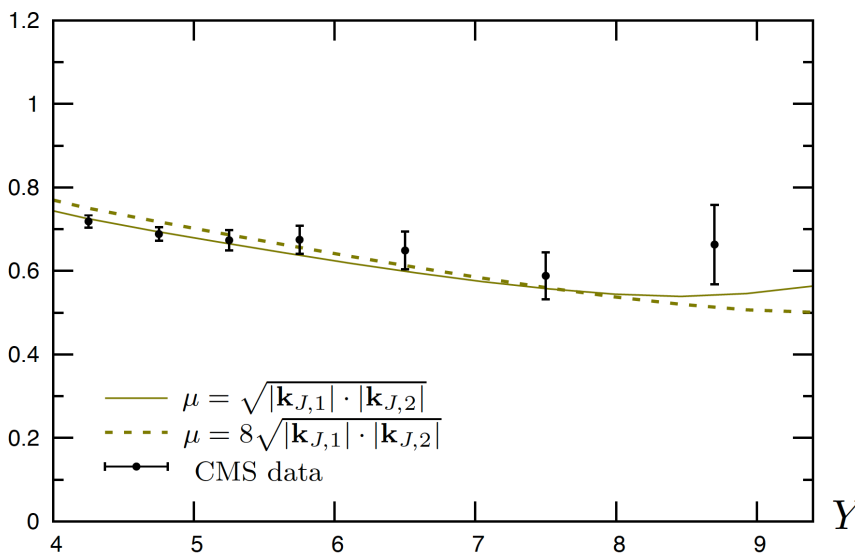

Fig. (7). Comparison of the full NLL BFKL calculation with either a "natural" scale $\mu=\sqrt{\left|\mathbf{k}_{J, 1}\right| \cdot\left|\mathbf{k}_{J, 2}\right|}$ or a large scale $\mu=8 \sqrt{\left|\mathbf{k}_{J, 1} \cdot\right| \cdot \mathbf{k}_{J, 2} \mid}$ and the CMS data, using symmetric cuts defined in Eq. (13). Left: value of $\langle\cos 3 \varphi\rangle$ as a function of the rapidity separation $Y$. Right: value of $\langle\cos 2 \varphi\rangle /\langle\cos \varphi\rangle$ as a function of the rapidity separation $Y$.

mixed treatment where the NLL Green's function is used together with LL vertices cannot describe the data. On the other hand, the results of our complete NLL calculation do not agree very well with the data when the scales involved, are fixed at their "natural" value $\sqrt{\left|\mathbf{k}_{J, 1}\right| \cdot\left|\mathbf{k}_{J, 2}\right|}$. The ratios of these observables are very stable with respect to changes of the scales and our calculation describes the data quite well.

The azimuthal distribution has also been measured by CMS, and its description based on our full NLL BFKL treatment with a natural renormalization/factorization scale fails for the nearside configurations. This description, as well as the description of $\langle\cos \varphi\rangle,\langle\cos 2 \varphi\rangle$ and $\langle\cos 3 \varphi\rangle$ is very successful provided one takes a large renormalization/factorization scale, much larger than the natural one. We have shown recently [25] that this can be understood when fixing the renormalization scale according to the physically motivated Brodsky-Lepage-Mackenzie procedure [26], in the spirit of [27, 28], which indeed leads to a very good description of the CMS data.

To find a fully conclusive evidence for the need of BFKLtype resummation in Mueller-Navelet jets, a comparison with a fixed order (NLO) treatment would be needed. We have compared our predictions with the ones obtained with the fixed order NLO code Dijet in an asymmetric configuration, as required to get stable results in a fixed order approach. We found that for the observables $\langle\cos n \varphi\rangle$ no significant difference is observed when taking into account the scale uncertainties. On the other hand, for $\langle\cos 2 \phi\rangle /\langle\cos \phi\rangle$ and $\langle\cos 3 \varphi\rangle /\langle\cos 2 \varphi\rangle$ the two calculations lead to noticeably different results. Since these observables are quite stable with respect to scale variations, they are well-suited to study resummation effects at high energy. We thus, believe that an experimental analysis with different lower cuts on the transverse momenta of the jets would be of great interest. 

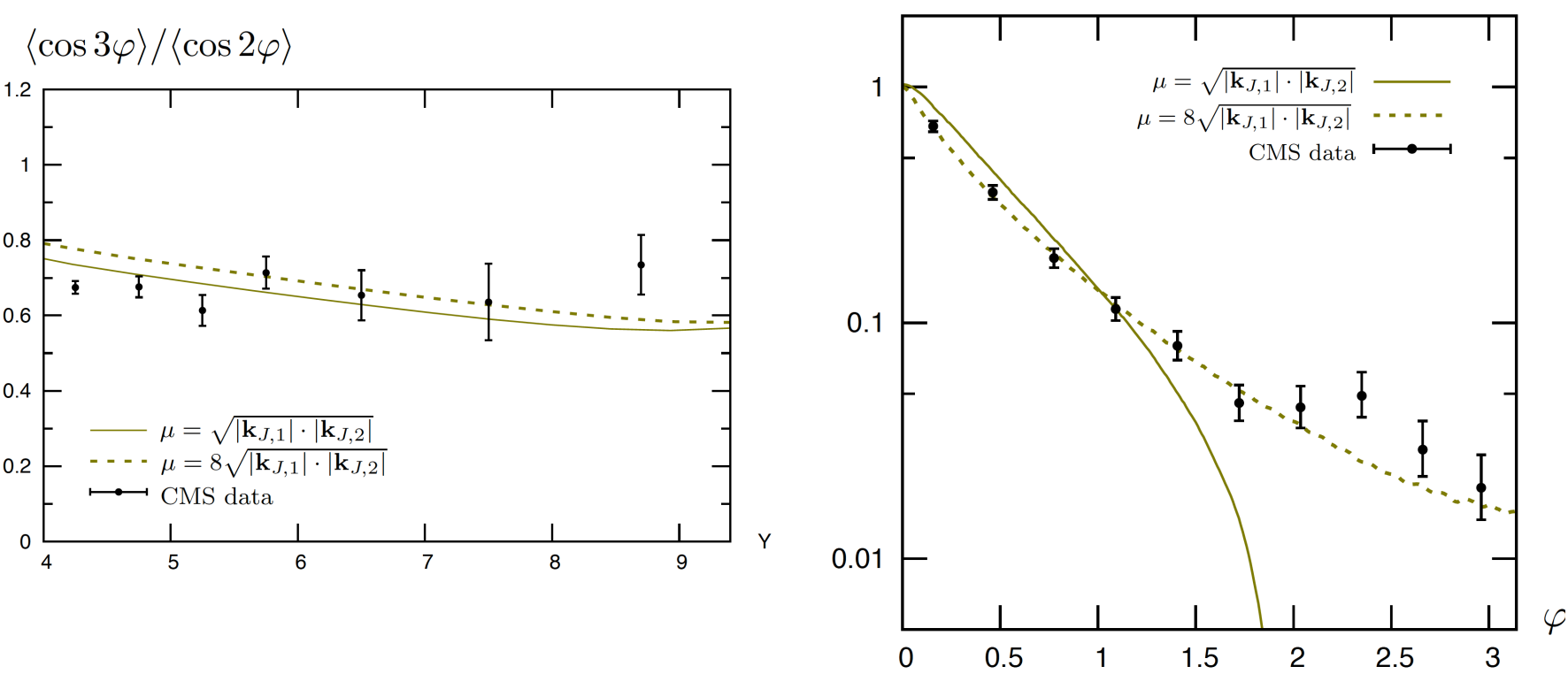

Fig. (8). Comparison of the full NLL BFKL calculation with either a "natural" scale $\mu=\sqrt{\left|\mathbf{k}_{J, 1}\right| \cdot\left|\mathbf{k}_{J, 2}\right|}$ or a large scale $\mu=8 \sqrt{\left|\mathbf{k}_{J, 1}\right| \cdot\left|\mathbf{k}_{J, 2}\right|}$ and the CMS data, using symmetric cuts defined in Eq. (13). Left: value of $\langle\cos 3 \varphi\rangle /\langle\cos 2 \varphi\rangle$ as a function of the rapidity separation $Y$. Right: value of the azimuthal distribution integrated over the range $6<Y<9.4$ as a function of $\varphi$.

\section{CONFLICT OF INTEREST}

The authors confirm that this article content has no conflict of interest.

\section{ACKNOWLEDGEMENTS}

We thank Michel Fontannaz, Cyrille Marquet and Christophe Royon for providing their codes and for stimulating discussions. We warmly thank Grzegorz Brona, David d'Enterria, Hannes Jung, Victor Kim and Maciej Misiura for many discussions and fruitful suggestions on the experimental aspects of this study.

We thank the organizers, the Tel Aviv French ambassy and the French CEA (IPhT and DSM) for support. This work is supported by the Joint Research Activity Study of Strongly Interacting Matter (HadronPhysics3, Grant Agreement no. 283286) under the 7th Framework Programme of the European Community and by the Polish Grant NCN No. DEC-2011/01/B/ST2/03915.

\section{REFERENCES}

(a). Askew AJ, Golec-Biernat KJ, Kwiecinski J, Martin AD, Sutton PJ. Implications of scaling violations of F2 at HERA for perturbative QCD. Phys Lett B 1994; 325: 212-8. (b). Navelet H, Peschanski R, Royon C, Wallon S. Proton structure functions in the dipole picture of BFKL dynamics. Phys Lett B 1996; 385: 357-64. (c). Munier S, Peschanski RB. High-energy factorization predictions for the charm structure function F2c at HERA. Nucl Phys B 1998; 524: 377-93. (d). Bartels J, De Roeck A, Lotter H. The $\gamma * \gamma *$ total cross section and the BFKL Pomeron at $\mathrm{e}+\mathrm{e}-$ colliders. Phys Lett B 1996; 389: 742-8. (e). Brodsky SJ, Hautmann F, Soper DE. Probing the QCD Pomeron in e+e- collisions. Phys Rev Lett 1997; 78: 803. (f). [Erratum-ibid. 79 (1997) 3544]. (g). Bialas A, Czyz W, Florkowski W. Total $\gamma * \gamma *$ cross section and the QCD dipole picture. Eur Phys J C 1998; 2: 683-9. (h). Boonekamp M, De Roeck A, Royon C, Wallon S. $\gamma * \gamma *$ total cross section in the dipole picture of BFKL dynamics. Nucl Phys B
1999; 555: 540-64. (i). Kwiecinski J, Motyka L. Probing the QCD pomeron in doubly tagged e+e- collisions. Phys Lett B 1999; 462: 203-10. (j). Brodsky SJ, Fadin VS, Kim VT, Lipatov LN, Pivovarov GB. The QCD pomeron with optimal renormalization. J Exp Theor Phys Lett 1999; 70: 155-60. (k). Brodsky SJ, Fadin VS, Kim VT, Lipatov LN, Pivovarov GB. High-energy QCD asymptotics of photon-photon collisions. J Exp Theor Phys Lett 2002; 76: 249-52.

[2] Mueller AH. Parton Distributions at very small x-values. Nucl Phys Proc Suppl 1991; 18C: 125-32.

[3] (a). Ginzburg IF, Panfil SL, Serbo VG. Semihard $\gamma \gamma \rightarrow$ V1V2, $\gamma \gamma \rightarrow \mathrm{V}+\mathrm{X}$ processes $(\mathrm{V}=\varrho 0, \omega, \phi, \gamma)$ : The possibility of the experimental investigation of the QCD pomeron. Nucl Phys B 1987; 284: 685-705. (b). Ginzburg IF, Panfil SL, Serbo VG. The semihard processes $\gamma \gamma \rightarrow \Psi X, \gamma \gamma \rightarrow \Psi \Psi, \gamma \gamma \rightarrow \varrho \Psi$. Nucl Phys B 1988; 296: 569-81. (c). Ryskin MG. Diffractive J / psi electroproduction in LLA QCD. Z Phys C 1993; 57: 89-92. (d). Mueller AH, Tang WK. High energy parton-parton elastic scattering in QCD. Phys Lett B 1992; 284: 123-6. (e). Ivanov DY, Kirschner R. Polarization in diffractive electroproduction of light vector mesons. Phys Rev D 1998; 58: 114026. (f). Ivanov DY, Kirschner R, Schafer A, Szymanowski L. Light vector meson photoproduction at large $t$. Phys Lett B 2000; 478: 101-13. (g). [Erratum-ibid B 2001; 498: 295]. (h). Pire B, Szymanowski L, Wallon S. Double diffractive rho-production in $\gamma * \gamma *$ collisions. Eur Phys J C 2005; 44: 545-58. (i). Enberg R, Pire B, Szymanowski L, Wallon S. BFKL resummation effects in $\gamma * \gamma *-->\rho \rho$. Eur Phys J C 2006; 45: 759-69. (j). [Erratum-ibid. C 2007; 51: 1015]. (k). Segond M, Szymanowski L, Wallon S. Diffractive production of two $\rho 0 \mathrm{~L}$ mesons in e+e-collisions. Eur Phys J C 2007; 52: 93 112. (1). Ivanov DY, Papa A. Electroproduction of two light vector mesons in the next-to-leading approximation. Nucl Phys B 2006; 7 32: 183-99. (m). Ivanov DY, Papa A. Electroproduction of two light vector mesons in the next-to-leading BFKL: Study of systematic effects. Eur Phys J C 2007; 49: 947-55. (n). Caporale F, Papa A, Vera AS. Collinear improvement of the BFKL kernel in the electroproduction of two light vector mesons. Eur Phys J C 2008; 53: 525-32.

[4] (a). Fadin VS, Kuraev EA, Lipatov LN. On the pomeranchuk singularity in asymptotically free theories. Phys Lett B 1975; 60: 50-2. (b). Kuraev EA, Lipatov LN, Fadin VS. Multi-reggeon processes in the Yang-Mills theory. Sov Phys J Exp Theor Phys 
1976; 44: 443-50. (c). Kuraev EA, Lipatov LN, Fadin VS. The pomeranchuk singularity in nonabelian gauge theories. Sov Phys J Exp Theor Phys 1977; 45: 199-204. (d). Balitsky II, Lipatov LN. The pomeranchuk singularity in quantum chromodynamics. Sov J Nucl Phys 1978; 28: 822-9.

[5] Mueller AH, Navelet $\mathrm{H}$. An inclusive minijet cross section and the bare pomeron in QCD. Nucl Phys B 1987; 282: 727-44.

[6] (a). Fadin VS, Lipatov LN. BFKL pomeron in the next-to-leading approximation. Phys Lett B 1998; 429: 127-34. (b). Ciafaloni M, Camici G. Energy scale(s) and next-to-leading BFKL equation. Phys Lett B 1998; 430: 349-54.

[7] (a). Bartels J, Colferai D, Vacca GP. The NLO jet vertex for Mueller-Navelet and forward jets: the quark part. Eur Phys J C 2002; 24: 83-99. (b). Bartels J, Colferai D, Vacca GP. The NLO jet vertex for Mueller-Navelet and forward jets: the gluon part. Eur Phys J C 2003; 29: 235-49.

[8] Caporale F, Ivanov DY, Murdaca B, Papa A, Perri A. The next-toleading order jet vertex for Mueller-Navelet and forward jets revisited. J High Energy Phys 2012; 1202: 101-28.

[9] (a). Duca VD, Schmidt CR. Dijet production at large rapidity intervals. Phys Rev D 1994; 49: 4510. (b). Stirling WJ. Production of jet pairs at large relative rapidity in hadron-hadron collisions as a probe of the perturbative pomeron. Nucl Phys B 1994; 423: 56-79.

[10] CMS Collaboration. Azimuthal angle decorrelations of jets widely separated in rapidity in pp collisions at $\sqrt{s}=7 \mathrm{TeV}$, CMS Physics Analysis Summary CMS-PAS-FSQ-12-002, 2013.

[11] Ducloué B, Szymanowski L, Wallon S. Confronting MuellerNavelet jets in NLL BFKL with LHC experiments at $7 \mathrm{TeV}$. J High Energy Phys 2013; 1305: 096.

[12] (a). Kotikov AV, Lipatov LN. NLO corrections to the BFKL equation in QCD and in supersymmetric gauge theories. Nucl Phys B 2009; 582: 19-43. (b). Kotikov AV, Lipatov LN. DGLAP and BFKL evolution equations in the $\mathrm{N}=4$ supersymmetric gauge theory. Nucl Phys B 2003; 661: 19-61. (c). [Erratum-ibid. B 2004;685: 405]. (d). Ivanov DY, Papa A. Electroproduction of two light vector mesons in the next-to-leading approximation. Nucl Phys B 2006; 732: 183. (e). Vera AS. The effect of NLO conformal spins in azimuthal angle decorrelation of jet pairs. Nucl Phys B 2006; 746: 1-14

[13] Sabio-Vera A, Schwennsen F. The azimuthal decorrelation of jets widely separated in rapidity as a test of the BFKL kernel. Nucl Phys B 2007; 776: 170-86.

[14] Schwennsen F. Phenomenology of jet physics in the BFKL formalism at NLO. 2007. [hep-ph/0703198]

[15] Colferai D, Schwennsen F, Szymanowski L, Wallon S. Mueller Navelet jets at LHC - complete NLL BFKL calculation. J High Energy Phys 2010; 1012: 026.

[16] Ivanov DY, Papa A. The next-to-leading order forward jet vertex in the small-cone approximation. J High Energy Phys 2012; 1205: 086 .
[17] Caporale F, Ivanov DY, Murdaca B, Papa A. Mueller-Navelet small-cone jets at LHC in next-to-leading BFKL. Nucl Phys B 2013; 877: 73-94.

[18] Caporale F, Murdaca B, Vera AS, Salas C. Scale choice and collinear contributions to Mueller-Navelet jets at LHC energies. Nucl Phys B 2013; 875: 134-51.

[19] Martin AD, Stirling WJ, Thorne RS, Watt G. Parton distributions for the LHC. Eur Phys J C 2009; 63: 189-285.

[20] (a). Salam GP. A resummation of large sub-leading corrections at small x. J High Energy Phys 1998; 07: 019. (b). Ciafaloni M, Colferai D. The BFKL equation at next-to-leading level and beyond. Phys Lett B 1999; 452: 372-8. (c). Ciafaloni M, Colferai D, Salam GP. Renormalization group improved small-x equation. Phys Rev D 1999; 60: 114036. (d). Ciafaloni M, Colferai D, Salam GP, Stasto AM. Renormalization group improved small-x Green's function. Phys Rev D 2003; 68: 114003.

[21] Marquet C, Royon C. Azimuthal decorrelation of Mueller-Navelet jets at the Tevatron and the LHC. Phys Rev D 2009; 79: 034028.

[22] Aurenche P, Basu R, Fontannaz M. Jet-jet and hadron-jet correlations in hadro- and electroproduction. Eur Phys J C 2008; 57: 681-8.

[23] Ducloué B, Szymanowski L, Wallon S. Mueller-Navelet jets at LHC: a clean test of QCD resummation effects at high energy? 2013. arXiv:1309.2672 [hep-ph].

[24] Ducloué B, Szymanowski L, Wallon S. Mueller-Navelet jets at LHC: The first complete NLL BFKL study. PoS QNP 2012; 2012: 165.

[25] Ducloué B, Szymanowski L, Wallon S. Evidence for high-energy resummation effects in Mueller-Navelet jets at the LHC. arXiv:1309.3229 [hep-ph]

[26] Brodsky SJ, Lepage GP, Mackenzie PB. On the elimination of scale ambiguities in perturbative quantum chromodynamics. Phys Rev D 1983; 28: 228.

[27] (a). Brodsky SJ, Fadin VS, Kim VT, Lipatov LN, Pivovarov GB The QCD pomeron with optimal renormalization. J Exp Theor Phys Lett 1999; 70: 155-60. (b). Brodsky SJ, Fadin VS, Kim VT, Lipatov LN, Pivovarov GB. High-energy QCD asymptotics of photon-photon collisions. J Exp Theor Phys Lett 2002; 76: 249-52. (c). [Pisma Zh Eksp Teor Fiz 2002; 76: 306].

[28] (a). Angioni M, Chachamis G, Madrigal JD, Vera AS. Dijet production at large rapidity separation in $\mathrm{N}=4$ supersymmetric Yang-Mills theory. Phys Rev Lett 2011; 107: 191601. (b). Hentschinski M, Vera AS, Salas C. Hard to soft pomeron transition in small-x deep inelastic scattering data using optimal renormalization. Phys Rev Lett 2013; 110: 041601. (c). Hentschinski M, Vera AS, Salas C. $F_{2}$ and $F_{L}$ at small $x$ using a collinearly improved BFKL resummation. Phys Rev D 2013; 87: 076005 . 